\section{Inhibition of Fusarium culmorum by Streptomyces albidoflavus}

THOUGH soil actinomycetes can sometimes elaborate antibiotics when grown in unamended sterile soil ${ }^{\mathbf{1}}$, it has usually been found necessary to enrich such soils with energy sources in order to demonstrate production of antibiotic ${ }^{2}$. In unsterile soil cultures, production of actinomycete antibiotics in clearly detectable amounts has only rarely been reported ${ }^{3}$. The following investigation was made to determine the ability of Streptomyces albidoflavus to inhibit the growth of the fungus Fusarium culmorum under a variety of nutritive conditions and particularly with small amounts of readily available carbohydrateconditions likely to be unfavourable for luxuriant growth of either organism.

When both organisms were seeded at a distance from each other on plates of mineral salt solution agar containing 1 per cent glucose, growth of the fungus towards the actinomycete was checked. With only 0.01 per cent glucose, the check was never complete and fungal hyphæ grew slowly but steadily towards the actinomycete. The effects of other sources of energy, more likely to be present in soil, were then tested. The actinomycete and the fungus were placed at a distance on plain agar buffered to

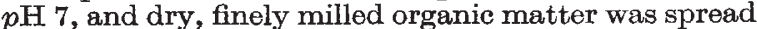
over each organism but not over the space between the organisms, so that growth of the fungus towards the actinomycete could be observed. On control plates without added organic material, growth of the fungus was slightly inhibited. Strong inhibition was obtained with dried grass. However, with wheat straw, roots, farmyard manure, leaf mould, peat and 'lignin', antagonism was even less than on the control plates with no added material. All these organic materials and clear extracts of them destroyed the antibiotic activity of actinomycete culture filtrates to which they were added. Presumably, on the agar plates, dried grass enabled the actinomycete to produce an amount of antibiotic in excess of that destroyed by it. Bentonite was very effective in removing antibiotic activity of actinomycete culture filtrates, presumably by adsorption. This effect was also exerted, though to a less extent, by other clays and by whole soils. Bentonite, when added to an agar medium, also strongly reduced antagonism between the actinomycete and the fungus.

When both organisms were grown together in sand culture, the addition of bentonite reduced but did not completely prevent inhibition of $F$. culmorum by the actinomycete. Thus, the actinomycete could inhibit the fungus by means other than antibiotic action.

A direct lysis of the contents of the fungal hyphæ by actinomycete mycelium in contact with it was observed in sand culture. This direct attack was prevented or greatly reduced by the presence of bentonite which, presumably, adsorbed the lytic agent. There was evidence that the actinomycete did not influence growth of the fungus in sterile soil either by antibiotic action or by direct attack, but that there was some competition between the organisms for nutrients or space.

In sand cultures there was a direct relation between the amount of glucose supplied and the antagonistic effect of the actinomycete. This relation was also found when bentonite was present, so that a high level of glucose presumably increased competition for limiting nutrients as well as influencing the production of antibiotic secretions. Lysis of the fungus was also most evident at high concentrations of glucose. Survival of both organisms together was favoured by low concentration of glucose. It would appear, therefore, that the conditions which favour the luxuriant development of an organism growing in pure culture may often be those that will tend to prevent its growth in association with others.

\section{F. A. SkInner}

Soil Microbiology Department,

Rothamsted Experimental Station,

Harpenden, Herts. July 29.

${ }^{1}$ Gottlieb, D., and Siminoff, P., Phytopath., 42, 91 (1952).

2 Nickell, L. G., and Burkholder, P. R., J, Amer. Soc. Agron., 39, 771 (1947).

s Gregory, K. F., et al., Amer. J. Bot., 39, 405 (1952).

\section{An Unknown Acid from Brain Extracts chromatographed on Silica Gel}

Since partition chromatography on silica gel allows the simultaneous quantitative determination of the individual acids of the polycarboxylic acid cycle ${ }^{1,2}$, it became of interest to apply this technique to the measurement of the organic acids in brain tissues. Fig. 1 shows the chromatographic pattern of the

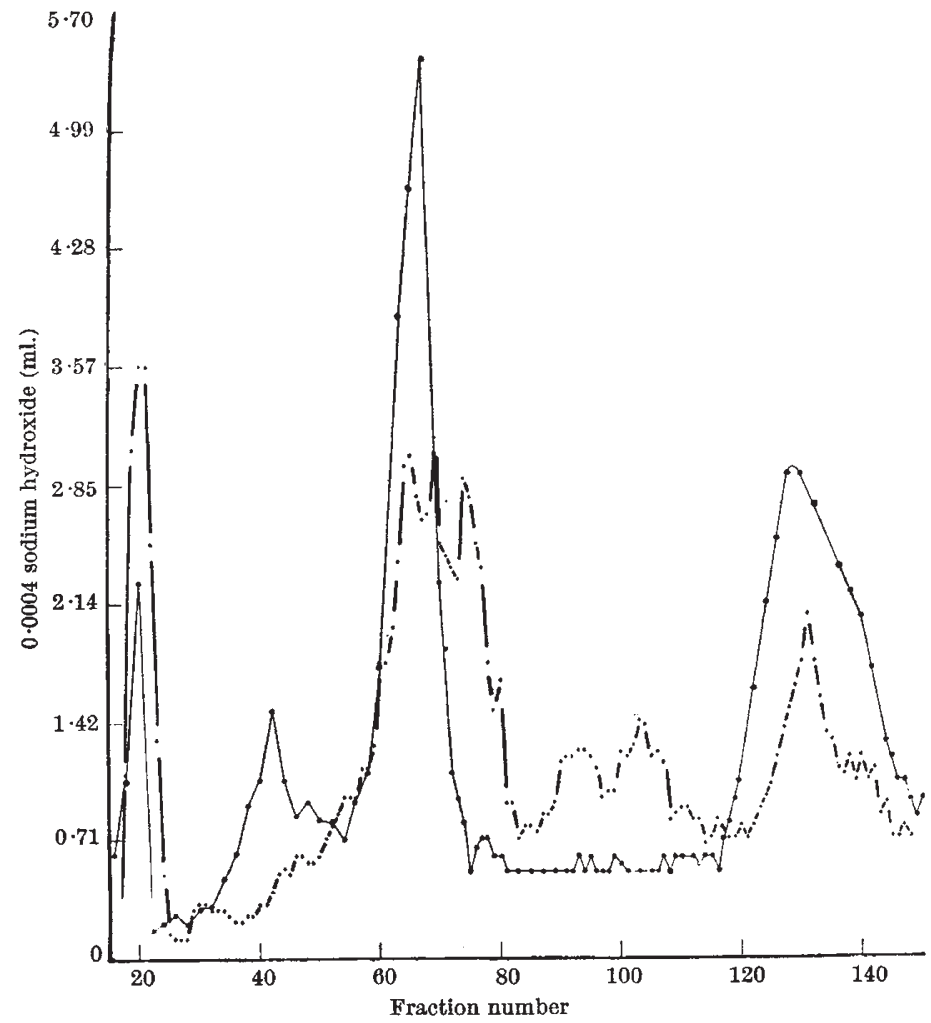

Fig. 1. Typical curves resulting when $1 \mathrm{gm}$ of brain tissue taken from man and turtle is chromatographed as under refs. 1 and 2 . Each fraction represents $1.5 \mathrm{ml}$. of effluent. The values for human brain tissue might be exaggerated because of anzesthesia used during the operation. Full line, human; broken line, turtle 